\title{
A fluorescence resonance energy transfer (FRET)-based redox sensor reveals physiological role of thioredoxin in the yeast Saccharomyces cerevisiae.
}

\section{$\operatorname{AUTHOR}(S)$ :}

Oku, Masahide; Hoseki, Jun; Ichiki, Yayoi; Sakai, Yasuyoshi

\section{CITATION:}

Oku, Masahide ... [et al]. A fluorescence resonance energy transfer (FRET)-based redox sensor reveals physiological role of thioredoxin in the yeast Saccharomyces cerevisiae. FEBS letters 2013, 587(6): 793-798

\section{ISSUE DATE:}

2013-03-18

\section{URL:}

http://hdl.handle.net/2433/173130

\section{RIGHT:}

(C) 2013 Federation of European Biochemical Societies. Published by Elsevier B.V.; This is not the published version. Please cite only the published version.; この論文は出版社版でありません。引用の際には 出版社版をご確認ご利用ください。 


\title{
A Fluorescence Resonance Energy Transfer (FRET)-based Redox Sensor Reveals Physiological \\ Role of Thioredoxin in the Yeast Saccharomyces cerevisiae
}

\author{
Masahide $\mathrm{Oku}^{1}$, Jun Hoseki ${ }^{1,2}$, Yayoi Ichiki ${ }^{1}$, and Yasuyoshi Sakai ${ }^{1,2,3}$
}

${ }^{1}$ Division of Applied Life Sciences, Graduate School of Agriculture and ${ }^{2}$ Research Unit for Physiological Chemistry, the Center for the Promotion of Interdisciplinary Education and Research, Kyoto University,

Kitashirakawa-Oiwake, Sakyo, Kyoto 606-8502, Japan

${ }^{3}$ CREST, Japan Science and Technology Agency, Kawaguchi Center Building, 4-1-8, Honcho, Kawaguchi-shi, Saitama 332-0012, Japan

To whom correspondence should be addressed: Yasuyoshi Sakai, Division of Applied Life Sciences,

Graduate School of Agriculture, Kyoto University, Kitashirakawa-Oiwake, Sakyo, Kyoto 606-8502, Japan, Tel:+81-75-753-6385; Fax: +81-65-753-6454; E-mail: ysakai@kais.kyoto-u.ac.jp 


\begin{abstract}
The physiological roles of the thioredoxin isozymes in the yeast Saccharomyces cerevisiae were investigated using a novel FRET-based redox probe, Redoxfluor. After establishing responsiveness of the probe toward thioredoxin, we followed the fluorescence signal of Redoxfluor expressed in the yeast and found that one of the thioredoxin isozymes, Trx2, was required for maintaining the redox status when stationary culture of the organism was exposed to starvation and mild-heat stresses. The failure to maintain redox balance under the tested condition preceded decreased viability of the trx 2 mutants, indicating the functional importance of the cytoplasmic thioredoxin in adaptation to environmental changes.
\end{abstract}

\title{
Keywords
}

FRET; redox regulation; thioredoxin; yeast physiology 


\section{Highlights}

Redoxfluor indicated reduced state in vitro by thioredoxin application.

Starvation and mild-heat stresses caused oxidized cytoplasmic state of trx2 mutants.

The oxidized state in the stress condition preceded viability loss of $\operatorname{tr} x 2$ mutants. 


\section{Introduction}

Maintenance of intracellular redox status is an important cellular activity, whose failure is associated with diverse pathological and aging processes [1,2]. To prevent undesirable accumulation of reactive oxygen species (ROS), eukaryotic cells possess diverse antioxidant enzymes such as catalase, superoxide dismutase, and peroxidases [3].

The reducing equivalents required for these enzymes are mainly derived from glutathione or thioredoxin. Glutathione, which is present at millimolar levels inside cells, supports a broad substrate spectrum of peroxidase and reductase activities [4]. Thioredoxins are also utilized as electron donors for a subset of antioxidant enzymes, especially for the peroxiredoxin family [5]. The yeast S. cerevisiae has two cytoplasmic thioredoxins, namely $\operatorname{Trx} 1$ and $\operatorname{Trx} 2$, and the transcriptional activity of the latter is induced by multiple environmental factors such as oxidative stress and growth state transition to the stationary phase [6,7]. The oxidized forms of the cytoplasmic thioredoxins are reduced back by a thioredoxin reductase Trr1 at the expense of NADPH.

To date, functional analyses of the yeast thioredoxins have been conducted mainly in the context of resistance to externally or experimentally added oxidative or reducing chemicals $[6,8]$, and little is known about the physiological contributions of the isozymes to other environmental conditions. Moreover, while double deletion of the cytoplasmic thioredoxin genes was reported to cause growth defects and severe sensitivity to chemical applications [9], no clear phenotype resulting from the loss of a single thioredoxin gene has been reported, except for the reduced resistance to hydrogen peroxide caused by deletion of TRX2 [7]. This is partly because of the lack of appropriate methods to monitor intracellular redox status reflecting the redox balance of the electron donor molecules as well as the ROS accumulation.

In a previous study, we devised a new redox sensor protein named Redoxfluor [10] by utilizing the redox-sensing region of Yap1. S. cerevisiae Yap1 is one of the major transcription factors that sense intracellular redox status [11-13]. Redoxfluor is comprised of a tandem repeat of the Yap1C-terminal region flanked by two fluorescent proteins, Cerulean and mCitrine. Changes in FRET efficiency between 
the two fluorescent proteins occur in response to various oxidative and reductive stimuli, indicating higher FRET rates during exposure to more reduced environments. This tendency contrasts with another FRET-based redox sensor protein [14] which shows lower FRET efficiencies under more reduced conditions. Notably, both of the FRET-based redox sensors also respond to changes in glutathione redox states in vitro, and thus can be used as sensors of the physiological redox status in vivo $[10,15]$. However, the response of Redoxfluor to another important redox compound, thioredoxin, remained to be investigated.

In this study we showed that Redoxfluor senses reducing activity of thioredoxin at physiological levels. By using this probe we examined impacts of the cytoplasmic thioredoxin gene disruptions on the redox status in vivo, which led to the discovery that TRX2 is necessary for maintaining the cytoplasmic redox status in response to environmental stresses induced by transfer of the cells to water at $37^{\circ} \mathrm{C}$. Since the oxidized status observed in the mutant strain correlated with subsequent loss of viability, the physiological function of Trx2 in redox maintenance was elucidated. 


\section{Materials and Methods}

\subsection{Strains and culture conditions}

All of the strains used in this study were derived from W303-1B as listed in Table I. For target gene disruptions, Schizosaccharomyces pombe HIS5, Candida glabrata LEU2 and TRP1 cassettes were amplified by PCR along with $40 \mathrm{bp}$ extensions homologous to the upstream and downstream regions of the target genes (for the latter two cassettes using BYP1419 and BYP1805 plasmids, provided by National BioResource Project-Yeast, Japan, as templates), and introduced into the parental strain (YMO300). The strains used for microscopic and viability analyses (YMO301 to 308) were converted to prototrophs. Strains were cultured at $30^{\circ} \mathrm{C}$ in YPD medium [2\% Bacto-Peptone (BD-Difco), $1 \%$ Yeast Extract (BD-Difco), and 2\% D-glucose]. To attain stable and uniform expression of Redoxfluor, we carried out genome-integration of its construct into URA3 locus. To obtain cells in the exponential growth phase, overnight cultures in YPD medium were diluted in fresh YPD medium with an $A_{610}$ of 0.3 , and cultured at $30^{\circ} \mathrm{C}$ until the $A_{610}$ of the culture reached 2.0. Stationary phase cultures were obtained by culturing in YPD medium at $30^{\circ} \mathrm{C}$ for $96 \mathrm{~h}$, when the $A_{610}$ of the culture exceeds 25 .

\subsection{Redoxfluor experiments in vitro}

Redoxfluor protein was expressed and purified, and all of the biochemical experiments were conducted under anaerobic conditions as described in the previous study [10]. The purified protein was diluted to 0.5 $\mathrm{mg} / \mathrm{ml}$ in Reaction Buffer (50 mM Hepes- $\mathrm{NaOH}, \mathrm{pH}$ 7.0, $0.14 \mathrm{M}$ potassium chloride, and $1 \mathrm{mM}$ EDTA), incubated with $1 \mathrm{mM} 4^{\prime}-4^{\prime}$-dipyridyl sulfide (DPS) solution at $37^{\circ} \mathrm{C}$ for $10 \mathrm{~min}$, and subjected to gel filtration on a PD-10 column (GE Healthcare). In order to prepare thiol-modified thioredoxin, recombinant Trx 2 protein in Reaction Buffer was incubated with $1 \mathrm{mM}$ DTT at $37^{\circ} \mathrm{C}$ for $30 \mathrm{~min}$, followed by addition of $10 \mathrm{mM}$ iodoacetamide (IAA), and further incubated at $37^{\circ} \mathrm{C}$ for $2 \mathrm{hrs}$, followed by gel filtration on a PD-10 column to remove free IAA. The reaction was initiated by mixing $0.15 \mu \mathrm{M}$ of the DPS-oxidized Redoxfluor, $0.4 \mathrm{mM}$ NADPH, $1 \mu \mathrm{M}$ of purified recombinant thioredoxin reductase (Trr1), $1 \mu \mathrm{M}$ of purified recombinant thioredoxin peroxidase (Tsa1), and the indicated concentrations of $\operatorname{Trx} 2$ in 
reaction buffer. After incubation of the reaction samples at $30^{\circ} \mathrm{C}$ for the indicated periods, emission spectra of the reaction samples were obtained by excitation at $405 \mathrm{~nm}$ wavelength with RF5300PC spectrofluorometer (Shimadzu, Japan). The ratio of acceptor/donor fluorescence intensity [I(acc)/I(don)] was calculated as summation of fluorescence emission intensities in the wavelength range from 524 to $526 \mathrm{~nm}$ (after subtraction of the corresponding background intensity values) divided by sum value in the range from 474 to $476 \mathrm{~nm}$ (after background subtraction ). Cross-linking experiments with free thiol residues inside Redoxfluor proteins to methoxy-poly (ethylene glycol)-maleimide (mPEG-maleimide, from Laysan Bio) were carried out as described previously [10].

\subsection{Visualization of Redoxfluor signal ratio by fluorescence microscopy}

Ratio of fluorescence intensities from Redoxfluor in the S. cerevisiae strains was visualized with modifications of the method described previously [10]. We used an Olympus IX-70 inverted microscope equipped with a UplanApo 40x objective (Olympus) and a CoolSNAP HQ2 CCD camera (Photometrics). All of the filters for the fluorescence acquisition were from Omega: XF1201 (436AF8) for the excitation filter, XF2034 (455DRLP) for the dichroic filter, XF3075 (480AF30) for the emission filter passing through fluorescence from Cerulean, and XF3079 (535AF26) emission filter for fluorescence from mCitrine. The exposure time for acquiring the image was set to $0.1 \mathrm{sec}$ with Uniblitz shutter system (Vincent Associates). In order to acquire the images through the filters for Cerulean and mCitrine fluorescence in the same field with a minimal time interval, the emission filters were mechanically exchanged in a filter wheel controlled with MAC5000 (Ludl Electronic Products). The images gained through the filters for Cerulean and mCitrine fluorescence were subjected to Ratio Image program in MetaMorph software (version 7.0, Universal Imaging).

\subsection{In vivo assessment of signal ratio from Redoxfluor}

In the images obtained through the filter for Cerulean fluorescence, more than 30 points were selected (1 point/ cell) as regions of interest (ROIs), each of which was located inside the cytoplasmic region with $5 \times 5$ pixel dimensions. The ROIs of the same position were allocated in the corresponding image through 
the filter for mCitrine fluorescence. The signal intensities of the ROIs were measured with the Region Measurement program in the MetaMorph software. After subtraction of values from blank ROIs (outside the cell region), the signal intensities of the ROIs in the images through the filter for mCitrine were divided by the corresponding intensities of those through the filter for Cerulean(from which blank ROIs had also been subtracted). Three independent experiments were done for the data acquisition. The calculated values were averaged and represented as fluorescence intensity ratio (FI ratio) of Redoxfluor.

\subsection{Determination of glutathione levels by LC-MS}

The yeast cells equivalent to $5 \mathrm{OD}_{610}$ units were collected and resuspended in $1 \mathrm{~mL}$ of pure methanol pre-chilled at $-80^{\circ} \mathrm{C}$. The suspension was transferred to liquid nitrogen and subsequently thawed on ice. After centrifugation at $20,000 \mathrm{xg}$ for $5 \mathrm{~min}$, the supernatant fraction was retrieved and the pellet fraction was again resuspended in $1 \mathrm{~mL}$ of the pre-chilled methanol, subjected to the freeze and thawing and centrifuged to obtain the supernatant fraction. These supernatant fractions were combined and freeze-dried, and re-suspended in $0.5 \mathrm{~mL}$ of $1 \%$ acetonitrile solution. The samples were applied to Prominence nano HPLC (Shimadzu) - 4000QTRAP Mass spectrograph system (AB SCIEX) equipped with Hydrosphere C18 column (YMC) to determine reduced and oxidized glutathione levels as well as the amount of leucine as an internal control. The $\Delta \mathrm{E}_{\mathrm{GSH}}$ values representing the difference in redox potential of glutathione between wild type and mutant strains are calculated as below:

$\Delta \mathrm{E}_{\mathrm{GSH}}=-\mathrm{RT} / 2 \mathrm{~F} \ln ($ Ratio of GSH content in the extract from a given mutant relative to that of wild-type strain $)^{2} /$ (Ratio of GSSG content in the extract from the mutant relative to that of the wild-type strain) R, Gas constant; T, absolute temperature (K); F, Faraday constant.

2.6. Viability assay

Cells in the stationary phase were collected and resuspended in water at $1 A_{610}$ concentration, and incubated aerobically at $37^{\circ} \mathrm{C}$. An aliquot of the sample was collected every 4 days and diluted 10,000-fold with fresh water. The diluted suspension $(100 \mu \mathrm{l})$ was plated onto a YPD plate, and incubated at $30^{\circ} \mathrm{C}$ for 2-3 days before counting the number of colonies. 


\section{Results}

\subsection{Redoxfluor has increased its ratio of acceptor/donor fluorescence intensity in the presence of} functional thioredoxin

The response of Redoxfluor to the reductive activity of thioredoxin was examined in vitro (Fig. 1). Pioneering studies on the molecular mechanisms of S. cerevisiae Yap1 and its Schizosaccharomyces pombe orthologue Pap1 activities demonstrated that the disulfide bond formations at their cysteine residues are mediated by thiol peroxidases (Orp1 and Tsa1 in S. cerevisiae and Tpx1 in S. pombe) [16-18], while cleavage of the bond is catalyzed by thioredoxin [19]. Based on these findings, the ratio of acceptor/donor fluorescence intensity $[\mathrm{I}(\mathrm{acc}) / \mathrm{I}(\mathrm{don})]$ of Redoxfluor was periodically determined after the probe protein was incubated with recombinant yeast thioredoxin $(\operatorname{Trx} 2)$ in the reaction mixture of Tsa1 and thioredoxin regeneration system. The incubation saw a gradual increase in $\mathrm{I}(\mathrm{acc}) / \mathrm{I}(\mathrm{don})$, which indicated a shift to more reduced status of Redoxfluor (Fig. 1A). Since this increase in the ratio was suppressed in the absence of thioredoxin, we reasoned that Redoxfluor was reduced by an electron transfer from $\operatorname{Trx} 2$.

Based on data from high-throughput determinations of yeast protein concentrations [20], the intra-cellular concentration of the cytoplasmic thioredoxins ( $\operatorname{Trx} 1$ and $\operatorname{Trx} 2)$ was estimated to sub-micromolar level, given that the volume of a yeast haploid cell is $70 \mu \mathrm{m}^{3}$. We examined whether Redoxfluor responses to Trx 2 at this physiological level. As a result, the ratio [I(acc)/I(don) $]$ was found elevated with increasing concentrations of Trx2 (Fig. 1B). Reduction of disulfide bonds in Redoxfluor by Trx 2 was biochemically confirmed by subsequent incubation of the reaction mixture with a thiol-modifying reagent of 5-KDa size, mPEG-maleimide (Fig. 1C). Immunoblot analysis of the probe protein after the reaction showed that Trx 2 application increased the highly modified population of the probe protein, reflecting increased thiol residues available for mPEG-maleimide modification. The Trx2 action on Redoxfluor was thiol-dependent, since the $\operatorname{Trx} 2$ derivative whose thiol residues had been 
masked with iodoacetamide (IAA) did not increase in I(acc)/I(don) of Redoxfluor (Fig. 1B), nor augment the mPEG-maleimide modification (Fig. 1C)

Our previous study had shown that Redoxfluor sensed the redox states of glutathione couples (GSH-GSSG) [10]. We then examined whether the probe protein response with thioredoxin was also detected in the presence of a physiological level of glutathione couple. Notably, $\mathrm{I}(\mathrm{acc}) / \mathrm{I}(\mathrm{don})$ rose with increasing concentrations of thioredoxin even in the presence of the glutathione redox couple (Fig. 1B). These in vitro data strongly suggest that Redoxfluor is capable of sensing the reducing activity of thioredoxin under physiological conditions.

\subsection{Loss of cytoplasmic thioredoxins caused no indication of oxidative shift by Redoxfluor under authentic} culture conditions

Next we examined whether loss of cytoplasmic thioredoxins altered the redox status in the yeast $S$. cerevisiae, using strains expressing Redoxfluor under TDH3 promoter regulation. The introduction of Redoxfluor did not affect the cell growth of each strain (data not shown). The wild-type and mutant strains lacking one or both of the cytoplasmic thioredoxins ( $\operatorname{Trx} 1$ and $\operatorname{Tr} x 2)$ were cultured in glucose (YPD) medium to exponential or stationary phase, and subjected to fluorescence microscopy for assessing fluorescence intensity ratios (FI ratios) of Redoxfluor which reflect the FRET efficiencies of the probe protein (Fig. 2). The image reconstituted from Redoxfluor fluorescence covered a large part of the whole cells except the vacuoles, which are represented as black or green circles, due to the absence of the intact probe protein in these organelles (Fig. 2A). Compared with exponentially growing cells, the cells in the stationary phase exhibited higher FI ratio in the cytoplasm, indicating a more reduced status in the stationary phase (Fig. 2A and 2B), which is consistent with metabolite profiles shown in a previous study [21]. No remarkable difference in the FI ratio of Redoxfluor was detected among the tested strains, regardless of the growth phase (Fig. 2A and 2B), suggesting that loss of the cytoplasmic thioredoxins did not affect the overall redox status under these culture conditions. 


\subsection{Loss of Trx2 leads to a more oxidized status of cells during incubation in water at $37^{\circ} \mathrm{C}$}

We hypothesized that the physiological function of the cytoplasmic thioredoxins might be to maintain redox status under environmentally stressful conditions. In order to test this notion, we transferred the stationary cultures of the strains expressing Redoxfluor to water, which gives rise to nutrient starvation, and/or incubation at $37^{\circ} \mathrm{C}$ (mild heat stress), both of which are plausible challenges to yeast cells in natural environments. Especially, heat stress is known to affect yeast intracellular pH [22], which is likely to influence the redox states of thiol moieties inside cells.

Microscopic assessment indicated that the cytoplasmic thioredoxin-null $(\operatorname{trx} 1 \Delta \operatorname{tr} x 2 \Delta)$ mutant underwent a marked drop in the FI ratio as a sign of oxidative redox status, when stationary phase cells were incubated at $37^{\circ} \mathrm{C}$ for 4 days, regardless of the incubation medium (Fig. 3A and 3B). Deletion of TRX1 alone caused a slight, not visually discernible decrease in the FI ratio when cells were transferred to water at $30^{\circ} \mathrm{C}$ or exposed to mild heat stress alone, and led to even higher FI ratio than the wild-type strain when treated with the combination of the stresses (Fig. 3A and 3B). In contrast, loss of TRX2 led to a remarkable decrease in FI ratio of Redoxfluor when the cells were transferred to water at $37^{\circ} \mathrm{C}$ (Fig. $3 \mathrm{~A}$ and 3B). In parallel with this observation, deletion of TRX2 significantly decreased glutathione levels in the cells transferred to water at $37^{\circ} \mathrm{C}$, which eventually gave rise to more oxidized state of glutathione in the TRX2-deleted strains (Fig. 3C). These data indicate that redox maintenance is impaired in the thioredoxin mutants under these stress conditions.

\subsection{The oxidized status in the TRX2-deletion strain precedes a decrease in its viability}

We next examined whether the observed oxidative status in the TRX2-deleted strain was associated with a physiological phenotype. For these experiments we followed the viabilities of the strains used for the microscopic study during prolonged incubation in water at $37^{\circ} \mathrm{C}$. The initial 4-day incubation under the stress conditions did not cause a significant decrease in the viabilities of the wild-type or the single 
TRX-deletion strains, but caused a severe loss of viability of the cytoplasmic thioredoxin-null $(\operatorname{trx} 1 \Delta \operatorname{trx} 2 \Delta)$ mutant (Fig. 3C). Importantly, further incubation up to 12 days resulted in a clear viability decrease in the TRX2-deletion strain than in the wild-type or TRX1-deletion strain (Fig. 4). These data together with the microscopic result strongly suggests that $\operatorname{Trx} 2$ function in the redox maintenance may contribute to supporting cell survival under the environmentally stressful conditions. 


\section{Discussion}

Biochemical analysis (Fig. 1) demonstrated the response of Redoxfluor to the reductive activity of the yeast thioredoxin Trx2. This response was also detected without addition of the peroxiredoxin (Tsa1) in the reaction mixture, but with much slower kinetics and to a lesser extent (data not shown). This implies that the reduction process of Redoxfluor is facilitated by Tsa1. A plausible explanation of the Tsa1 role in the Redoxfluor response is that Tsa1 transiently forms a disulfide bond with Redoxfluor and the electron transfer from thioredoxin to the probe protein is mediated by the bound Tsa1 molecule, in analogy to the involvement of the peroxiredoxins in the regulations of Yap1 and Pap1activities [16-18],

The responsiveness of Redoxfluor toward thioredoxin, in combination with other previously-reported characteristics of this probe protein [10], enables us to visualize integrated redox status encompassing the redox states of both glutathione and thioredoxin as well as intra-cellular ROS levels. Although another redox sensor protein, roGFP2, was reported to have little response toward human Trx in the minute time scale $[23,24]$, it is possible that the steady-state signal gained from the probe protein may also be affected by thioredoxin.

Cells under normal culture conditions did not show remarkable differences in FI ratio of Redoxfluor depending on the presence of the TRX genes (Fig. 2), although transcriptional induction of TRX2 in the stationary phase was reported previously [7]. In these situations, activities of other redox-related enzymes such as glutathione dependent peroxidases, glutaredoxins, and catalases may be sufficient to maintain the redox status, making the effect of TRX gene disruptions undetectable. In a previous study, double deletion of TRX1 and TRX2 was found to cause constitutive activation of Yap1 activity, leading to induction of genes encoding redox-related enzymes [9]. Such a feedback system may be responsible for the redox maintenance in the absence of cytoplasmic thioredoxins.

The mutant strains lacking either of the cytoplasmic thioredoxins exhibited distinct indications of redox status from Redoxfluor, when the cells were exposed to both starvation and mild-heat stresses (in water at $37^{\circ} \mathrm{C}$ ): while the TRX2-deleted strain showed more oxidized status than the wild-type strain, the 
TRX1-deleted mutant showed more reduced status (Fig.3). One plausible hypothesis for explaining this result is that TRX1 deletion may up-regulate expressions of TRX2 or other anti-oxidant enzymes. Future studies addressing interrelationship between thioredoxin expressions and those of other redox-related enzymes will be necessary for the overall understanding of redox regulation.

In this study we aimed to reveal the function of thioredoxin in a physiological context, and chose to expose the cells to water and/or mild heat stress. Incubation of cells in water is a widely-used method to determine yeast chronological life span [25], and a previous study included the temperature shift to $37^{\circ} \mathrm{C}$ in the life-span assay to accelerate the decline in cell viability [26]. Thus, based on the data of this study (Fig. 4), Trx 2 could be defined as a positive factor for extending longevity of the yeast. This notion is consistent with recent findings that peroxiredoxin proteins (acceptors of electron from thioredoxin) are important for establishing longevity in several experimental systems [27], and hence the physiological importance of thioredoxin will be unveiled to a greater extent in relation to longevity. 


\section{Acknowledgements}

The authors thank Dr. Shusuke Kuge, Tohoku Pharmaceutical University, Japan, for his kind gift of the plasmids encoding yeast thioredoxin, thioredoxin peroxidase and thioredoxin reductase. This work was supported by MEXT/JSPS Grants-in-Aid for Scientific Research (24780100 to MO), (23770158 to JH), (11016060 to YS), and by JST, CREST. 


\section{REFERENCES}

[1] Terman, A. and Brunk, U.T. (2004). Aging as a catabolic malfunction. Int J Biochem Cell Biol 36, 2365-75.

[2] Zhang, Y., Du, Y., Le, W., Wang, K., Kieffer, N. and Zhang, J. (2011). Redox control of the survival of healthy and diseased cells. Antioxid Redox Signal 15, 2867-908.

[3] Herrero, E., Ros, J., Belli, G. and Cabiscol, E. (2008). Redox control and oxidative stress in yeast cells. Biochim Biophys Acta 1780, 1217-35.

[4] Grant, C.M. (2001). Role of the glutathione/glutaredoxin and thioredoxin systems in yeast growth and response to stress conditions. Mol Microbiol 39, 533-41.

[5] Kondo, N., Nakamura, H., Masutani, H. and Yodoi, J. (2006). Redox regulation of human thioredoxin network. Antioxid Redox Signal 8, 1881-90.

[6] Kuge, S. and Jones, N. (1994). YAP1 dependent activation of TRX2 is essential for the response of Saccharomyces cerevisiae to oxidative stress by hydroperoxides. EMBO J 13, 655-64.

[7] Garrido, E.O. and Grant, C.M. (2002). Role of thioredoxins in the response of Saccharomyces cerevisiae to oxidative stress induced by hydroperoxides. Mol Microbiol 43, 993-1003.

[8] Trotter, E.W. and Grant, C.M. (2002). Thioredoxins are required for protection against a reductive stress in the yeast Saccharomyces cerevisiae. Mol Microbiol 46, 869-78.

[9] Izawa, S., Maeda, K., Sugiyama, K., Mano, J., Inoue, Y. and Kimura, A. (1999). Thioredoxin deficiency causes the constitutive activation of Yap1, an AP-1-like transcription factor in Saccharomyces cerevisiae. J Biol Chem 274, 28459-65.

[10] Yano, T., Oku, M., Akeyama, N., Itoyama, A., Yurimoto, H., Kuge, S., Fujiki, Y. and Sakai, Y. (2010). A novel fluorescent sensor protein for visualization of redox states in the cytoplasm and in peroxisomes. Mol Cell Biol 30, 3758-66.

[11] Kuge, S., Jones, N. and Nomoto, A. (1997). Regulation of yAP-1 nuclear localization in response to oxidative stress. EMBO J 16, 1710-20. 
[12] Lee, J., Godon, C., Lagniel, G., Spector, D., Garin, J., Labarre, J. and Toledano, M.B. (1999). Yap1 and Skn7 control two specialized oxidative stress response regulons in yeast. J Biol Chem $274,16040-6$.

[13] Okazaki, S., Tachibana, T., Naganuma, A., Mano, N. and Kuge, S. (2007). Multistep disulfide bond formation in Yap1 is required for sensing and transduction of $\mathrm{H} 2 \mathrm{O} 2$ stress signal. Mol Cell $27,675-88$.

[14] Kolossov, V.L., Spring, B.Q., Sokolowski, A., Conour, J.E., Clegg, R.M., Kenis, P.J. and Gaskins, H.R. (2008). Engineering redox-sensitive linkers for genetically encoded FRET-based biosensors. Exp Biol Med (Maywood) 233, 238-48.

[15] Lin, C. et al. (2011). Imaging in real-time with FRET the redox response of tumorigenic cells to glutathione perturbations in a microscale flow. Integr Biol (Camb) 3, 208-17.

[16] Delaunay, A., Isnard, A.D. and Toledano, M.B. (2000). H2O2 sensing through oxidation of the Yap1 transcription factor. EMBO J 19, 5157-66.

[17] Bozonet, S.M., Findlay, V.J., Day, A.M., Cameron, J., Veal, E.A. and Morgan, B.A. (2005).

Oxidation of a eukaryotic 2-Cys peroxiredoxin is a molecular switch controlling the transcriptional response to increasing levels of hydrogen peroxide. J Biol Chem 280, 23319-27.

[18] Tachibana, T., Okazaki, S., Murayama, A., Naganuma, A., Nomoto, A. and Kuge, S. (2009). A major peroxiredoxin-induced activation of Yap1 transcription factor is mediated by reduction-sensitive disulfide bonds and reveals a low level of transcriptional activation. J Biol Chem 284, 4464-72.

[19] Kuge, S., Arita, M., Murayama, A., Maeta, K., Izawa, S., Inoue, Y. and Nomoto, A. (2001). Regulation of the yeast Yap1p nuclear export signal is mediated by redox signal-induced reversible disulfide bond formation. Mol Cell Biol 21, 6139-50.

[20] Ghaemmaghami, S., Huh, W.K., Bower, K., Howson, R.W., Belle, A., Dephoure, N., O'Shea, E.K. and Weissman, J.S. (2003). Global analysis of protein expression in yeast. Nature 425, 737-41. 
[21] Drakulic, T., Temple, M.D., Guido, R., Jarolim, S., Breitenbach, M., Attfield, P.V. and Dawes, I.W. (2005). Involvement of oxidative stress response genes in redox homeostasis, the level of reactive oxygen species, and ageing in Saccharomyces cerevisiae. FEMS Yeast Res 5, 1215-28.

[22] Imai, T. and Ohno, T. (1995). The relationship between viability and intracellular $\mathrm{pH}$ in the yeast Saccharomyces cerevisiae. Applied and Environmental Microbiology 61, 3604-8.

[23] Gutscher, M., Pauleau, A.L., Marty, L., Brach, T., Wabnitz, G.H., Samstag, Y., Meyer, A.J. and Dick, T.P. (2008). Real-time imaging of the intracellular glutathione redox potential. Nat Methods $5,553-9$.

[24] Meyer, A.J. and Dick, T.P. (2010). Fluorescent protein-based redox probes. Antioxid Redox Signal 13, 621-50.

[25] Fabrizio, P. and Longo, V.D. (2007). The chronological life span of Saccharomyces cerevisiae. Methods Mol Biol 371, 89-95.

[26] Piper, P.W., Harris, N.L. and MacLean, M. (2006). Preadaptation to efficient respiratory maintenance is essential both for maximal longevity and the retention of replicative potential in chronologically ageing yeast. Mech Ageing Dev 127, 733-40.

[27] Labunskyy, V.M. and Gladyshev, V.N. (2012). Role of Reactive Oxygen Species-Mediated Signaling in Aging. Antioxid Redox Signal 


\section{FIGURE LEGENDS}

FIGURE 1. Redoxfluor response to thioredoxin in vitro. A. Time course of change in the ratio of acceptor/donor fluorescence intensity of Redoxfluor after addition of thioredoxin. Purified and pre-oxidized Redoxfluor protein in a reaction mixture (See Materials and Methods) was incubated with (closed circle) or without (open circle) $5 \mu \mathrm{M}$ of recombinant $\operatorname{Tr} x 2$ at $30^{\circ} \mathrm{C}$ for the indicated time periods. The ratio value $[\mathrm{I}(\mathrm{acc}) / \mathrm{I}(\mathrm{don})]$ was calculated as described in Materials and Methods. The error bars indicate standard deviations from 3 independent experiments. $B$. Plots of the ratio of acceptor/donor fluorescence intensity versus applied thioredoxin concentrations. The purified and pre-oxidized Redoxfluor protein was incubated with the designated concentrations of thioredoxin, either intact or pre-treated with iodoacetamide (IAA) at $30^{\circ} \mathrm{C}$ for $2 \mathrm{~h}$. The reaction with the intact thioredoxin was also performed with $2.5 \mathrm{mM}$ reduced and $0.25 \mathrm{mM}$ oxidized glutathione in the mixture. The error bars indicate standard deviations. $C$. Modification of Redoxfluor with mPEG-maleimide after the application of thioredoxin. The reaction mixture used in $B$ was incubated with the thiol-modifying reagent mPEG-maleimide and subjected to immunoblot analysis for the detection of Redoxfluor. The positions of molecular weight markers (MW) are also shown. The asterisks show band positions of Redoxfluor modified with mPEG-maleimide.

FIGURE 2. Visualization of fluorescence intensity ratio of Redoxfluor expressed in the yeast cytoplasm. $A$. The indicated yeast strain expressing Redoxfluor was cultured in YPD medium to either exponential (EXP) or stationary (STA) phase, and subjected to fluorescence microscopy. The ratio value (FI ratio) reflecting the FRET efficiency of Redoxfluor in the cytosol was calculated from for each point as described in Materials and Methods, and visualized with the designated color gradation. (Note that ratios in the vacuoles were not included in the calculation.) WT, wild type. Bar, $10 \mu \mathrm{m}$. B. Comparison of the average FI ratios gained and averaged from 90cells (1 point per cell). The error bars indicate standard errors of the mean value. 
FIGURE 3. Thioredoxin contributions to maintenance of redox status. A. The stationary phase strains used in Fig. 2 were kept in the same medium or transferred to water, and incubated at either $30^{\circ} \mathrm{C}$ or $37^{\circ} \mathrm{C}$ (mild heat stress) for 4 days. Then the microscopic analysis was done as described in Fig. $2 A$. Bar, $10 \mu \mathrm{m}$. $B$. The averaged FI ratios were shown as in Fig. $2 B$. The error bars show standard errors of the mean. $C$. The cells after4-day incubation in water at $37^{\circ} \mathrm{C}$ were retrieved and subjected to determination of glutathione levels. The relative amounts of reduced (GSH) and oxidized (GSSG) glutathione were compared among the denoted strains. The error bar indicates standard deviations. The differences in the redox potential of glutathione $\left(\Delta \mathrm{E}_{\mathrm{GSH}}\right)$ between wild-type and the mutant and sample were also shown below the graph, along with their standard errors in parenthesis. The positive values indicate more oxidized state of glutathione in the denoted mutant strains. Note that the redox potential of glutathione depends on $[\mathrm{GSH}]^{2} /[\mathrm{GSSG}]$, not $[\mathrm{GSH}] /[\mathrm{GSSG}]$, and hence the decrease in GSH concentration gives rise to oxidized redox potential even when the ratio ([GSH]/[GSSG]) is unchanged.

FIGURE 4. TRX2 is required for maintenance of viability during incubation in water at $37^{\circ} \mathrm{C}$. The numbers of viable cells for the designated strains after the transfer to water and incubation at $37^{\circ} \mathrm{C}$ were plotted. The error bars show standard deviations. 
Table I S. cerevisiae strains $^{\text {a }}$ used in this study

\author{
Strain Name Genotype \\ YMO300 MATalpha ADE2 can1-100 his3-11 leu2-3,112 trp1-1,15, URA3::pRFY-1A \\ ( $\mathrm{P}_{T D H 3}-$ Redoxfluor- $\mathrm{T}_{T D H 3}$ in pRS306) \\ YMO301 YMO300, HIS3:: $\quad$ pRS303; LEU2::pRS305; TRP1::pRS304 \\ YMO302 YMO300, trx1 $\quad$ Y :: $($ Cg)TRP1; HIS3:: pRS303; LEU2::pRS305 \\ YMO303 YMO300, $\operatorname{trx} 2 \Delta::$ SpHIS5 (equivalent to ScHIS3); LEU2::pRS305; TRP1::pRS304 \\ YMO304 YMO300, trx1 I :: (Cg)TRP1; trx2A::SpHIS5; LEU2::pRS305
}

${ }^{a}$ All of the listed strains were generated in this study. 
A

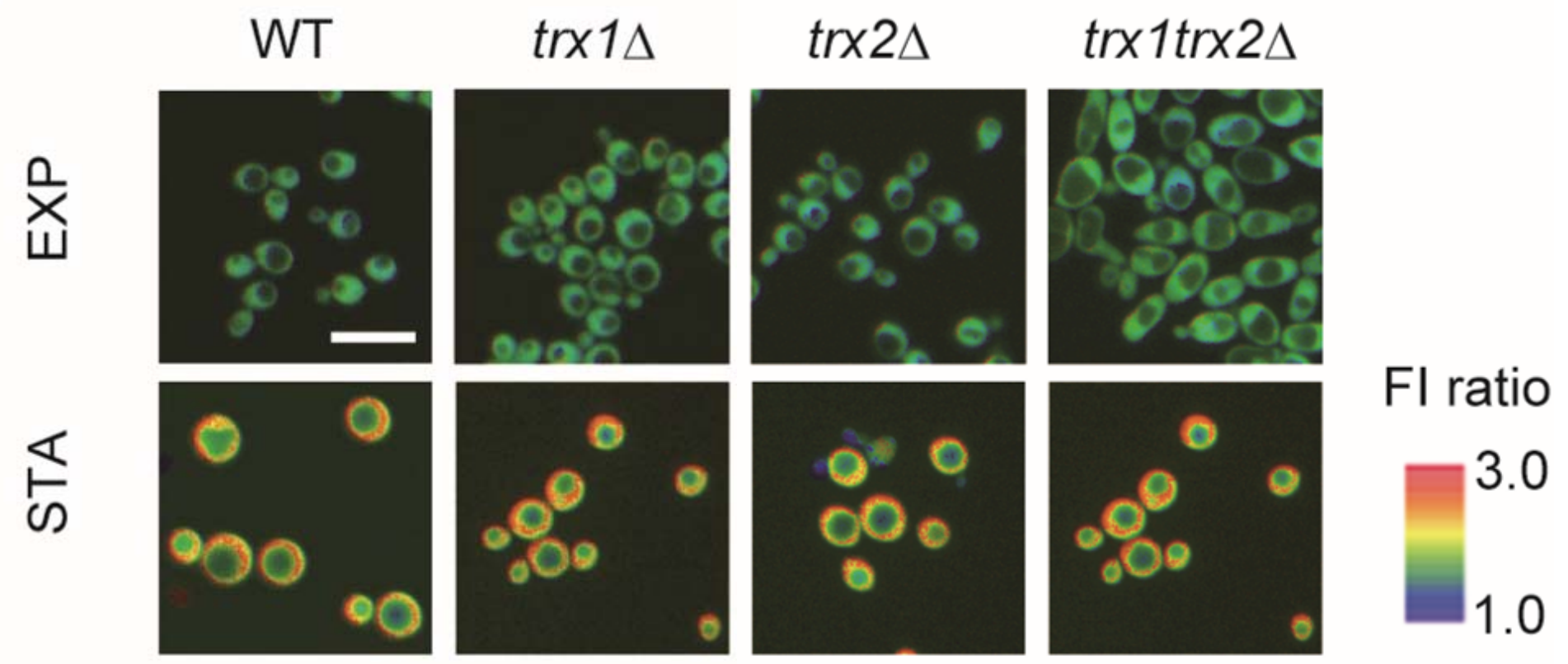

B

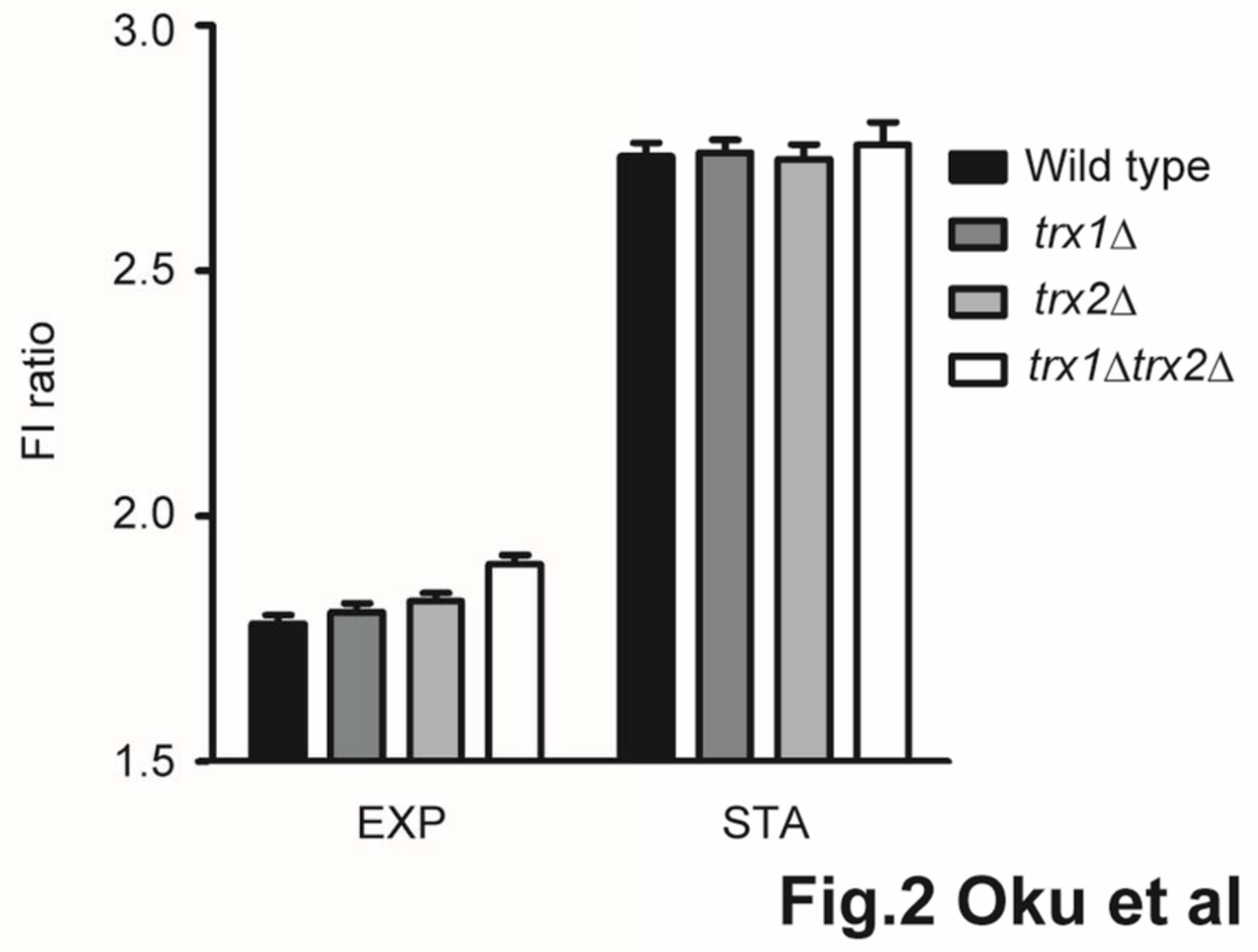


A

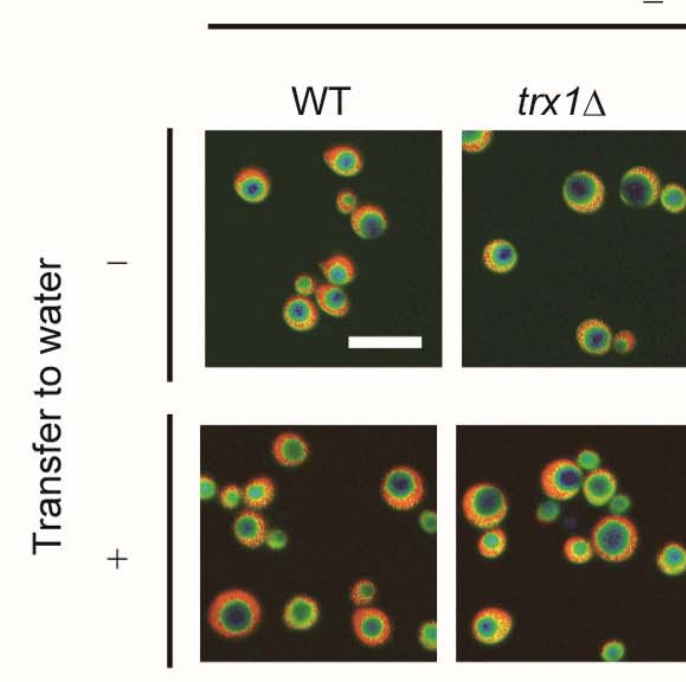

B
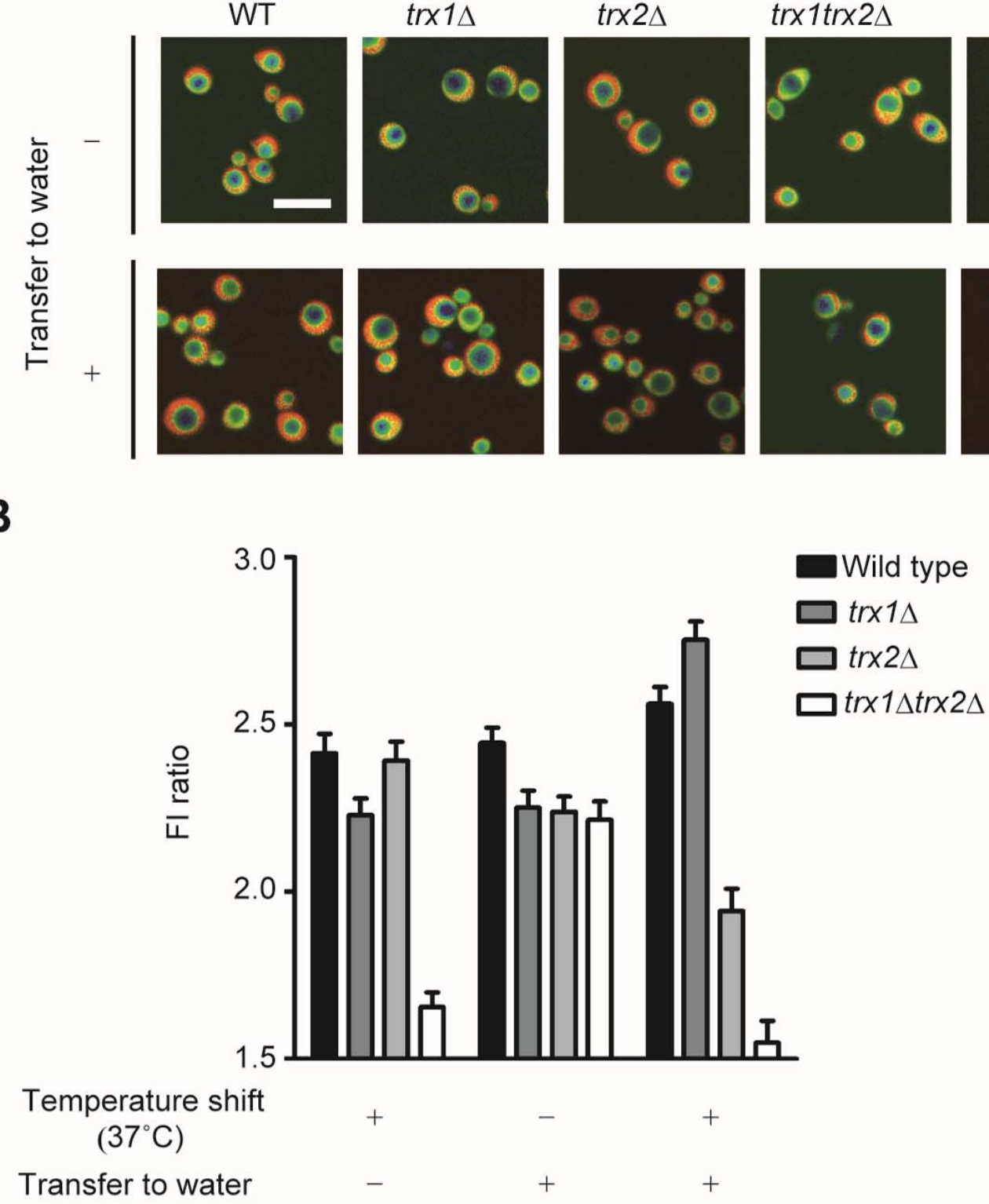
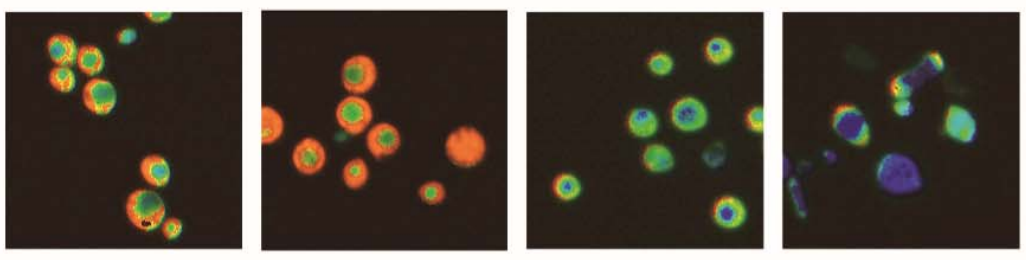

FI ratio

3.0

C

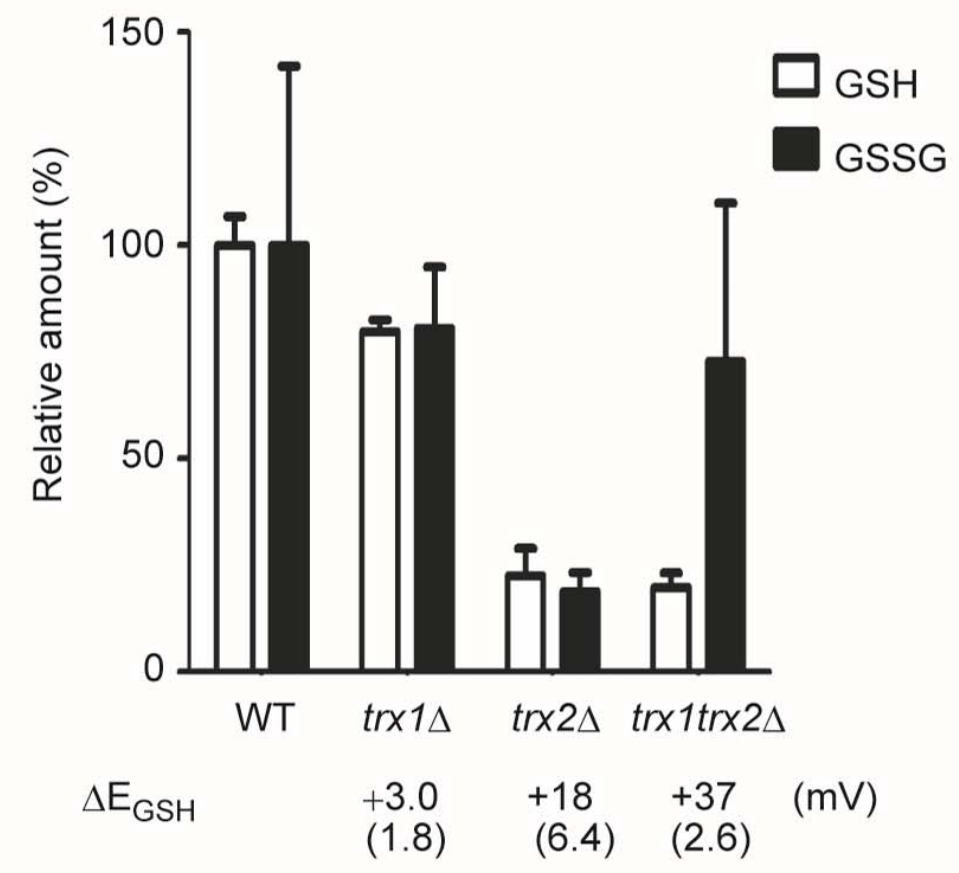

Fig. 3 Oku et al 


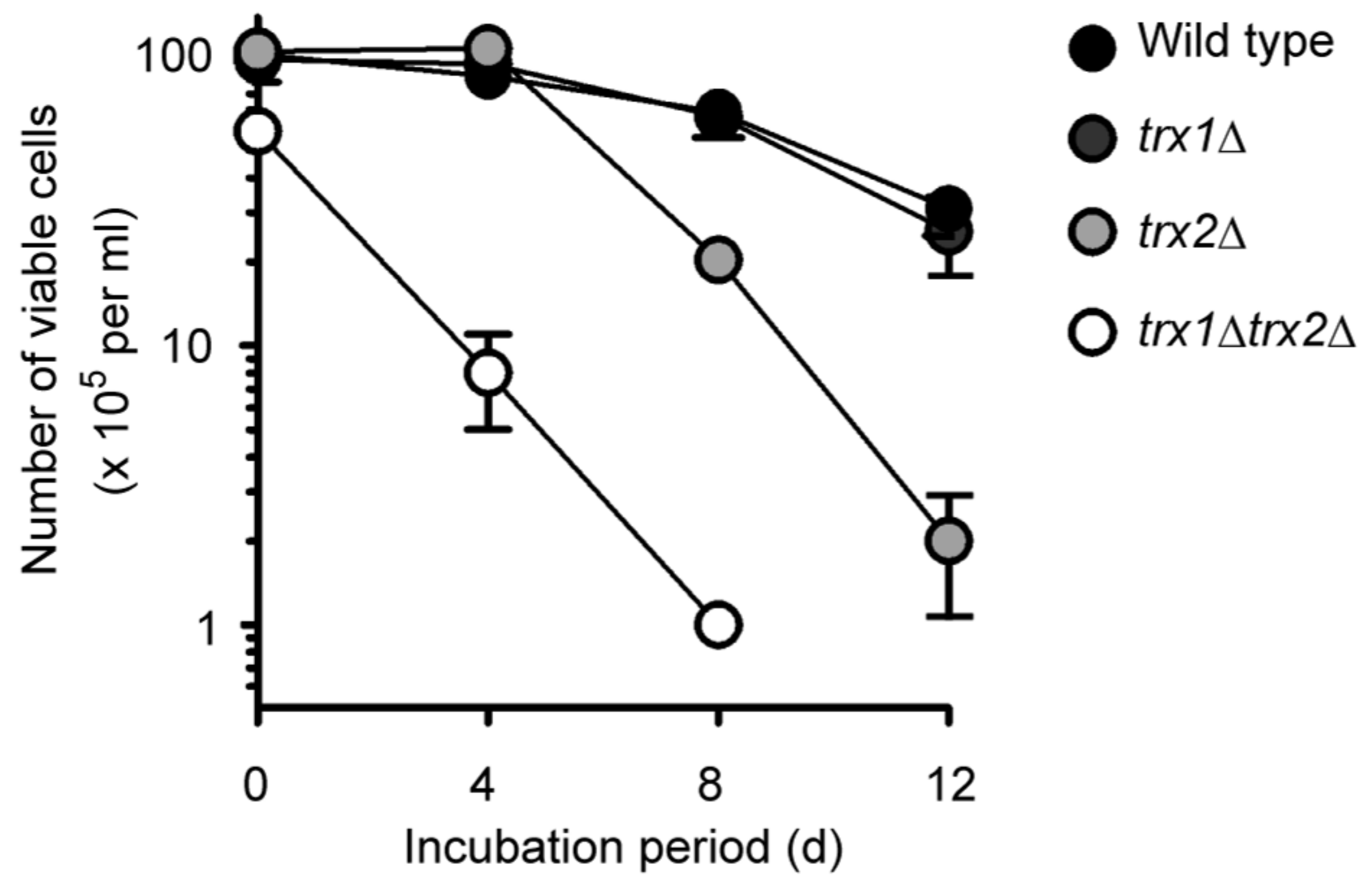

Fig. 4 Oku et al 
A

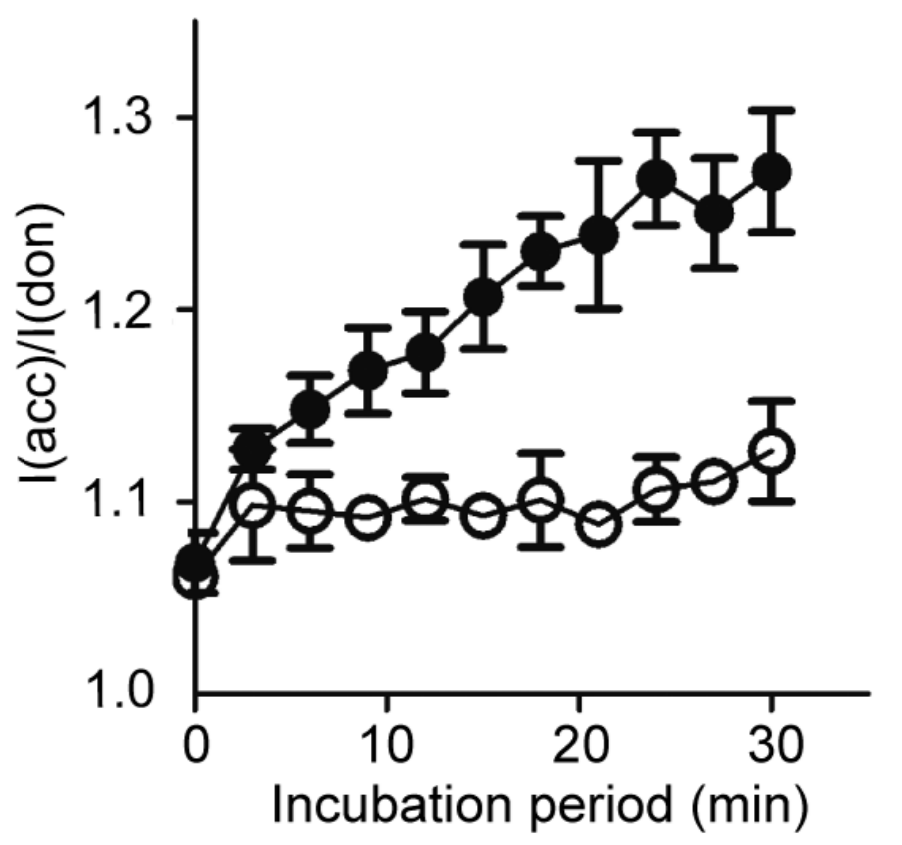

+ Thioredoxin

O No thioredoxin

B

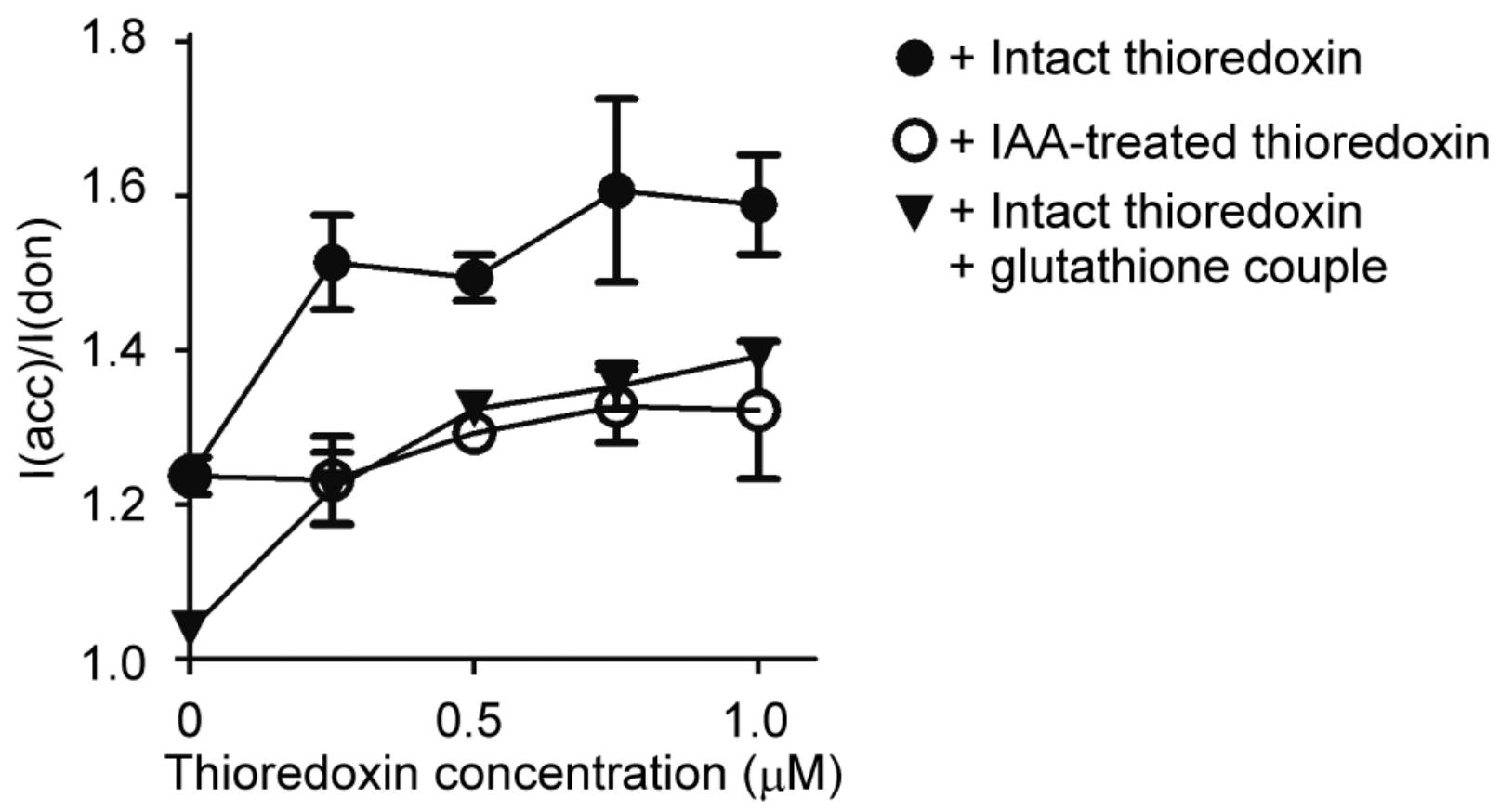

c

Thioredoxin $(\mu \mathrm{M})$

$0 \quad \frac{\text { Intact }}{0.51 .0} \frac{\text { IAA-treated }}{0.51 .0} \mathrm{MW}$

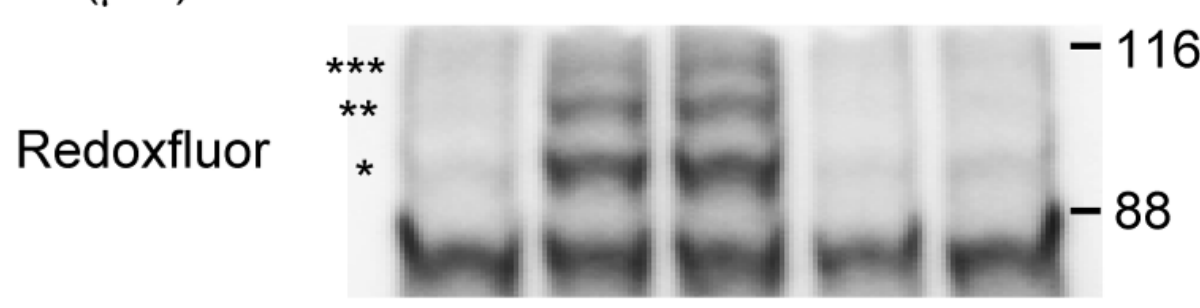

Fig. 1 Oku et al 